\title{
Diseño y elaboración de utensilios biodegradables a partir de la fibra del tallo de banano (Musa paradisiaca) como alternativa de uso para mitigar impactos ambientales causados por el plástico
}

Design and elaboration of biodegradable utensils from the fiber of the banana stem (Musa paradisiaca) as an alternative of use to mitigate environmental impacts caused by the plastic

Santiago Andrés Aguiar Conya. ${ }^{1}$, Marlene Jaqueline García Veloz. ${ }^{2} \&$ Sonia Mercedes Vallejo Abarca. ${ }^{3}$

Recibido: 12-11-2019 / Revisado: 30-11-2019 /Aceptado: 29-12-2019/ Publicado: 04-01-2020

\begin{abstract}
DOI: https://doi.org/10.33262/cienciadigital.v4i1.1118

Due to environmental requirements, today we choose to find new sources of renewable resources, to apply them in various areas, therefore, we have seen the need to produce biodegradable utensils, being an effective alternative to mitigate the use of conventional equipment whose characteristics have a very high degradation time. The research took place in the Industrial Processes laboratory of the Faculty of Science of the Polytechnic School of Chimborazo, the main raw material that was used was obtained from the banana stem to which purification and bleaching was done with $\mathrm{NaOH}$ at $30 \%$, $\mathrm{NaClO}$ and water that allowed the hardening of the fibers obtained, the average initial weight of the raw material without drying $(0.1454 \mathrm{Kg})$ was considered, the organic fibers were distributed in four drying trays which were subjected to the drying equipment of trays at a temperature of $50{ }^{\circ} \mathrm{C}$ for 7 hours once the fiber moisture was extracted, an average weight
\end{abstract}

1 Escuela Superior Politécnica de Chimborazo, Facultad de Ciencias, Riobamba, Ecuador, santiago.aguiar@espoch.edu.ec

2 Escuela Superior Politécnica de Chimborazo, Facultad de Ciencias, Riobamba, Ecuador, marlene.garcia@espoch.edu.ec

3 Escuela Superior Politécnica de Chimborazo, Facultad de Ciencias, Riobamba, Ecuador, sonia.vallejo@espoch.edu.ec 
of the dry raw material of $(0.04028 \mathrm{Kg})$ was obtained, eliminating $77.99 \%$ of moisture and then going to the process spraying in a ball mill turning the previously dried organic fibers into powder, then with the powder obtained the utensils were made by moistening the dry fibers to shape them. The fibers obtained achieved excellent mechanical strength, then an analysis was performed by infrared IR spectroscopy, presenting characteristics similar to those of a synthetic plastic and differentiating it by its easy degradation. The processing of the raw material for the elaboration of utensils does not cause environmental contamination becoming biodegradable waste after its use.

Keywords: Environmental, Biodegradable, Pollution, Fiber, Degradation.

\section{Resumen}

Debido a los requerimientos ambientales, hoy en día se opta por encontrar nuevas fuentes de recursos renovables, para aplicarlas en diversas áreas, por ello, se ha visto la necesidad de elaborar utensilios biodegradables, siendo una alternativa eficaz para mitigar el uso de enseres convencionales cuyas características presentan un tiempo de degradación muy elevado. La investigación tuvo lugar en el laboratorio de Procesos Industriales de la Facultad de Ciencias de la Escuela Superior Politécnica de Chimborazo, la materia prima principal que se utilizó se obtuvo a partir del tallo de banano al que se hizo una purificación y blanqueamiento con $\mathrm{NaOH}$ al 30\%, $\mathrm{NaClO}$ y agua que permitió el endurecimiento de las fibras obtenidas, se consideró el peso inicial promedio de la materia prima sin secar $(0,1454$ $\mathrm{Kg}$ ), las fibras orgánicas se distribuyeron en cuatro bandejas de secado las cuales fueron sometidas al equipo secador de bandejas a una temperatura de 50 grados centígrados durante 7 horas una vez extraída la humedad de las fibras se obtuvo un peso promedio de la materia prima seca de $(0,04028 \mathrm{Kg})$ lográndose eliminar el 77,99 \% de humedad para luego pasar al proceso de pulverización en un molino de bolas convirtiendo en polvo las fibras orgánicas previamente secadas, luego con el polvo obtenido se elaboraron los utensilios humedeciendo las fibras secas para dar la forma de los mismos. Las fibras obtenidas alcanzaron una excelente resistencia mecánica, luego se realizó un análisis mediante espectroscopia infrarroja IR, presentando características similares a las de un plástico sintético y diferenciándolo por su fácil degradación. El procesamiento de la materia prima para la 
elaboración de utensilios no causa contaminación ambiental convirtiéndose en desechos biodegradables luego de su uso.

Palabras claves: Ambiental, Biodegradable, Contaminación, Fibra, Degradación.

\section{Introducción}

Debido a la masiva producción y exportación de banano se han generado desechos provenientes de este sin ningún valor económico comercial para el sector agrícola de esta manera hemos visto la necesidad de encontrar una nueva forma de utilización de estos desechos con el propósito de eliminar la problemática ambiental y socioeconómica de un sector generalmente olvidado en nuestro país Ecuador. (Chivrac. F, 2009)

Conrad. U, 2006 y de acuerdo a bibliografía consultada se pudo constatar que posee características similares a las presentadas por otros desechos orgánicos entre estos las cascaras de coco, caña de azúcar entre otros.

De acuerdo a informes presentados por la Organización de las Naciones Unidas para la Agricultura y Alimentación (FAO) (2007) donde indica que Ecuador cubre un 25\% de las exportaciones anualmente de banano a nivel mundial.

Según afirmaciones del sector agrícola bananero se enuncia que los tallos generados durante el proceso de corte del producto listo para su comercialización, no poseen ningún valor o utilidad hasta este momento debido a la falta de investigación sobre el uso de estos, colocándonos de esta manera como los pioneros en la utilización y desarrollo de nuevas técnicas alternativas y viables del cuidado ambiental y generación de recursos económicos adicionales para este sector solventando el PIB de nuestro país.

El consumismo y sistema económico actual sumados a la capacidad que tiene el hombre para transformar el medio en productos de corta duración, fabricados con materiales de difícil descomposición y reciclado, han provocado numerosos problemas ambientales.

En una investigación realizada aseguran que la utilización de plástico es considerada como la tercera aplicación del petróleo más utilizada a nivel mundial siendo consumido anualmente 300 millones de toneladas.

La problemática fundamental es la inclusión en las legislaciones ambientales el manejo de los residuos provenientes del sector agrícola ganadero.

Los desechos que originamos deben ser tratados de manera adecuada para que no contaminen más aún los desechos generados de este importante sector productivo de nuestro país como es el agro. 
Las administraciones públicas y la industria, así como otros sectores de la sociedad, siguen generando una gran cantidad de residuos que han ido aumentando año tras año durante las últimas décadas.

Esta situación ha provocado que la generación y gestión de los residuos constituya un reto ambiental para las sociedades modernas y uno de los principales obstáculos para lograr un desarrollo sostenible verdadero. Un problema de tal dimensión y complejidad requiere de diferentes soluciones. Existen numerosas alternativas técnicas y tecnológicas disponibles para mejorar los sistemas de producción industrial y gestión de los residuos, jugando un papel fundamental la implicación social y la participación ciudadana.

En términos legales actualmente los productores de envases tienen que cumplir con ciertas restricciones a las que se enfrentan que los obligará a hacerse cargo de sus residuos y de los embalajes usados

Una de estas alternativas es la aplicación de conceptos como la biodegradabilidad que es la capacidad que tienen las sustancias y los materiales orgánicos de descomponerse en sustancias más sencillas debido a la actividad enzimática de microorganismos.

Cuando se completa el proceso biológico, se obtiene la transformación total de las sustancias orgánicas iniciales en moléculas inorgánicas simples como agua, dióxido de carbono y metano.

El fenómeno de la biodegradación forma parte del ciclo natural de la vida en la tierra, basado en el carbono. Gracias a la actividad de fotosíntesis de plantas y algas, y a la inagotable energía solar, el dióxido de carbono de la atmósfera se absorbe para sintetizar los azúcares y las demás sustancias utilizadas por los vegetales para crecer y desarrollarse. A través de la cadena alimentaria, el flujo de materia y de energía pasa de las plantas a los herbívoros y de estos a los carnívoros.

Cuando los organismos vegetales y animales mueren, los microorganismos -presentes siempre en el medio ambiente- se nutren del material orgánico mediante procesos de biodegradación que liberan agua y dióxido de carbono a la atmosfera, cerrándose así el ciclo.

Imitando y valorizando estos procesos naturales, también los residuos orgánicos de las actividades humanas pueden eliminarse mediante la biodegradación. Para que esta operación sea eficaz es necesario identificar el ambiente ideal en el que este fenómeno puede ser maximizado y es preciso definir un tiempo de duración del proceso que sea industrializable y compatible con los ritmos de producción de estos residuos orgánicos. 
En la naturaleza cada residuo orgánico tiene sus tiempos de degradación, paja y madera emplean más tiempo que almidón y celulosa. De la misma manera, en ambientes fríos y secos los procesos de biodegradación son más lentos que en ambientes cálidos y húmedos.

Esto significa que la biodegradación depende mucho de la naturaleza química de la sustancia o materia que se desea biodegradar y del ambiente de biodegradación. Los ambientes en los que la biodegradación se produce a un buen ritmo y que permiten una gestión industrial son los del compostaje y de la digestión anaeróbica.

En estos sistemas, por consiguiente, se pueden tratar los residuos sólidos orgánicos, incluidos los procesados (por ejemplo, de plástico biodegradable) que tienen una velocidad de biodegradación compatible con estos tratamientos. En el caso del compostaje se obtendrá un compost maduro (que es un fertilizante) y en el caso de la digestión anaeróbica (seguida de estabilización en compostaje) se obtendrán biogases (y por consiguiente energía) y compost.

Otro ambiente biológicamente activo es el suelo: algunos materiales se pueden biodegradar totalmente en el suelo y esta propiedad puede aprovecharse en aplicaciones específicas como por ejemplo en el acolchado (cubrimiento del suelo).

Hasta la actualidad se han desarrollado un gran número de trabajos e investigación encaminados a reducir las cantidades de residuos plásticos generados y fabricar productos menos agresivos con el medio ambiente y que representan una amenaza potencial para muchos ecosistemas empleando conceptos de biodegradabilidad.

Por lo que nuestra investigación en la que propone mitigar en gran mediada la contaminación ambiental con la utilización de un residuo proveniente de la cáscara del banano considerado como un residuo orgánico y su empleo en la fabricación de utensilios en remplazo de los plásticos que se emplean para servir y almacenar alimentos.

\section{Metodología}

La experimentación se realizó en el laboratorio de Operaciones Unitarias de la Escuela Superior Politécnica de Chimborazo, aplicando las respectivas medidas de control antes durante y después de la elaboración de los utensilios biodegradables se realizaron las pruebas de resistencia mecánica para luego mediante espectroscopia infrarroja IR caracterizar el producto obtenido.

\section{Procedimiento Experimental}

Luego de la recolección de la materia prima se hizo un lavado, clasificación con la finalidad de garantizar un producto de buena calidad de los utensilios a obtener; se redujo y endurecieron las 
fibras aplicando un método de secado por bandejas en el que las fibras se sometieron a $50{ }^{\circ} \mathrm{C}$ durante 7 horas, se hizo una purificación y blanqueamiento con $\mathrm{NaOH}$ al 30\%, $\mathrm{NaClO}$ agua, para el endurecimiento de las fibras y lográndose eliminar la mayor cantidad de agua posible hasta tal punto que pueda pulverizarse para que los utensilios puedan ser moldeados, Se realizaron pruebas de resistencia de materiales y se hicieron análisis de laboratorio que corroboran que se obtuvo un producto de buena calidad.

A continuación, se describe el diagrama de flujo del proceso para la transformación de la materia prima en un material biodegradable para la elaboración de utensilios.

Figura 1: Diagrama de Flujo del Proceso

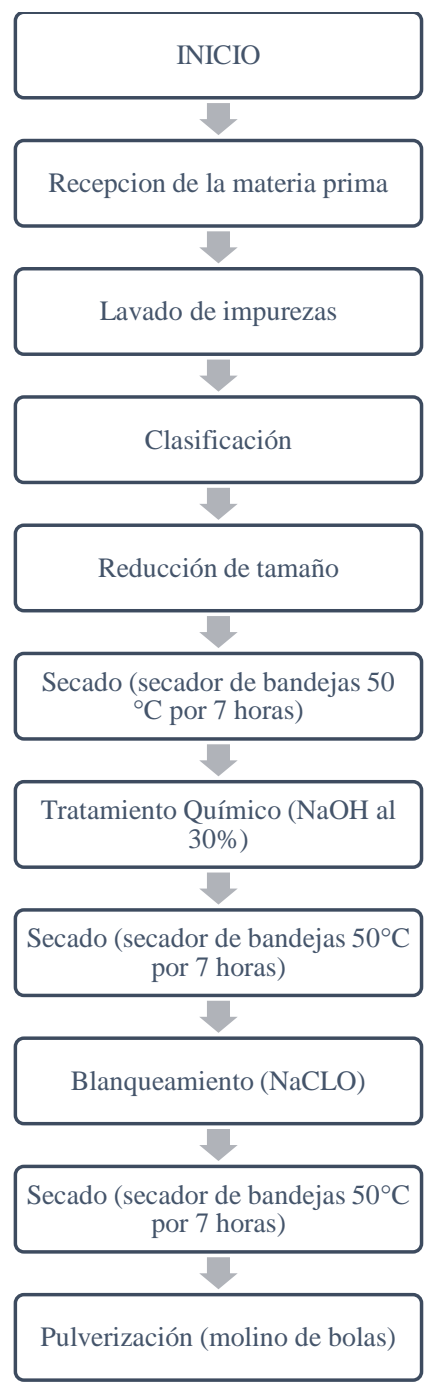

Fuente: Aguiar Santiago. 2020 


\section{Resultados}

La materia prima principal que se utilizó se obtuvo a partir del tallo de banano conocida como fibra de musa es una de las fibras naturales más fuertes del mundo. Biodegradable, compuesta principalmente de celulosa, hemicelulosas y lignina y su capacidad de la rotación, finura y resistencia a la tracción son factores analizados que determinan la calidad de las fibras obtenidas. Las fibras más gruesas y resistentes se toman de las vainas externas de los árboles de banano, mientras que las vainas interiores dan como resultado fibras más suaves a dichas fibras se les hizo una purificación y blanqueamiento con $\mathrm{NaOH}$ al 30\%, $\mathrm{NaClO}$ y agua que permitió el endurecimiento de las fibras obtenidas, se consideró el peso inicial promedio de la materia prima sin secar $(0,1454 \mathrm{Kg})$ como factor determinante de pérdida de humedad en los pasos subsiguientes del proceso, las fibras orgánicas se distribuyeron en cuatro bandejas de secado las cuales fueron sometidas al equipo secador de bandejas a una temperatura de $50{ }^{\circ} \mathrm{C}$ durante 7 horas tiempo en el cual se determinó el punto exacto en que las fibras pueden pasar a la siguiente etapa del proceso, una vez extraída la humedad de las fibras se obtuvo un peso promedio de la materia prima seca de (0,04028 Kg) lográndose eliminar el 77,99 \% de humedad para luego pasar al proceso de pulverización en un molino de bolas con el objeto de hacer polvo a las fibras obtenidas. En la tabla 1 se muestran los datos experimentales que se tomaron como base investigativa.

Tabla 1. Datos experimentales

\begin{tabular}{|c|c|c|c|c|c|}
\hline & $\begin{array}{l}\text { Peso inicial materia } \\
\text { prima (sin secar, } \mathrm{kg} \text { ) }\end{array}$ & $\begin{array}{l}\text { Peso materia prima } \\
(\text { seca, } \mathrm{kg})\end{array}$ & $\%$ de Humedad & $\begin{array}{l}\text { Peso neto de } \\
\text { platos }(\mathrm{kg})\end{array}$ & $\begin{array}{l}\text { Peso neto } \\
\text { vasos }(\mathrm{kg})\end{array}$ \\
\hline Bandeja 1 & 0,1454 & 0.0345 & 76.26 & $8.5 \times 10^{-3}$ & $3.3 \times 10^{-3}$ \\
\hline Bandeja 2 & 0.1818 & 0.040 & 77.99 & $8.5 \times 10^{-3}$ & $3.3 \times 10^{-3}$ \\
\hline Bandeja 3 & 0.2272 & 0.0488 & 78.52 & $8.5 \times 10^{-3}$ & $3.3 \times 10^{-3}$ \\
\hline Bandeja 4 & 0.1818 & 0.0378 & 79.20 & $8.5 \times 10^{-3}$ & $3.3 \times 10^{-3}$ \\
\hline PROMEDIO & 0,1454 & 0,04028 & 77,99 & $8.5 \times 10^{-3}$ & $3.3 \times 10^{-3}$ \\
\hline
\end{tabular}

Fuente: Aguiar Santiago. 2020

En base a los datos obtenidos experimentalmente se pudo identificar la viabilidad de este proyecto, puesto que por cada parte del tallo nos da como resultado una cantidad de 8 utensilios entre vasos y paltos, el peso neto promedio de los platos obtenidos es de $8.5 \times 10^{-3}$ por $\mathrm{Kg}$ que se traducen a una dimensión de platos de $156 \mathrm{~mm}$ x $14 \mathrm{~mm}$ y peso neto promedio de los vasos de $3.3 \times 10^{-3}$ por Kg traducido a dimensiones de vasos de $69 \mathrm{~mm}$ x $81 \mathrm{~mm}$ estos con una capacidad de $175 \mathrm{ml}$, esto muestra que este proyecto es de vital importancia para el sector agrícola puesto que significa un 
aumento en la economía de estos, según afirmaciones del sector agrícola bananero se enuncia que los tallos generados durante el proceso de corte del producto listo para su comercialización, no poseen ningún valor o utilidad hasta este momento debido a la falta de investigación sobre el uso de estos, colocándonos de esta manera como los pioneros en la utilización y desarrollo de nuevas técnicas alternativas y viables del cuidado ambiental y generación de recursos económicos adicionales para este sector solventando el PIB de nuestro país, cabe mencionar que este proyecto fue tratado con gente del sector quien nos indicó que el precio por tallo sería de 25 centavos de dólar Americano dejando una rentabilidad para abonos y costos de funda para la protección del fruto lo suficientemente conveniente.

Figura 2: Utensilios obtenidos

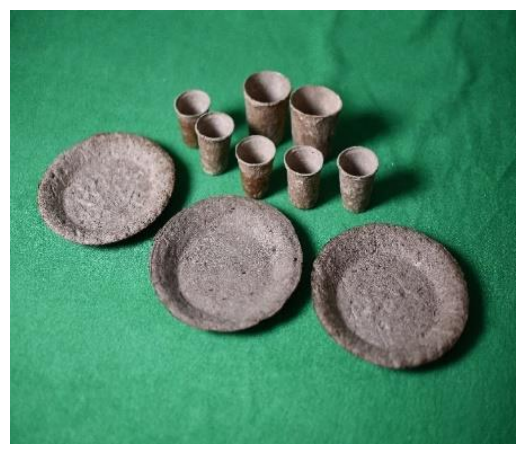

Fuente: Aguiar Santiago. 2020

Figura 3: Resultados de Espectrofotómetro IR

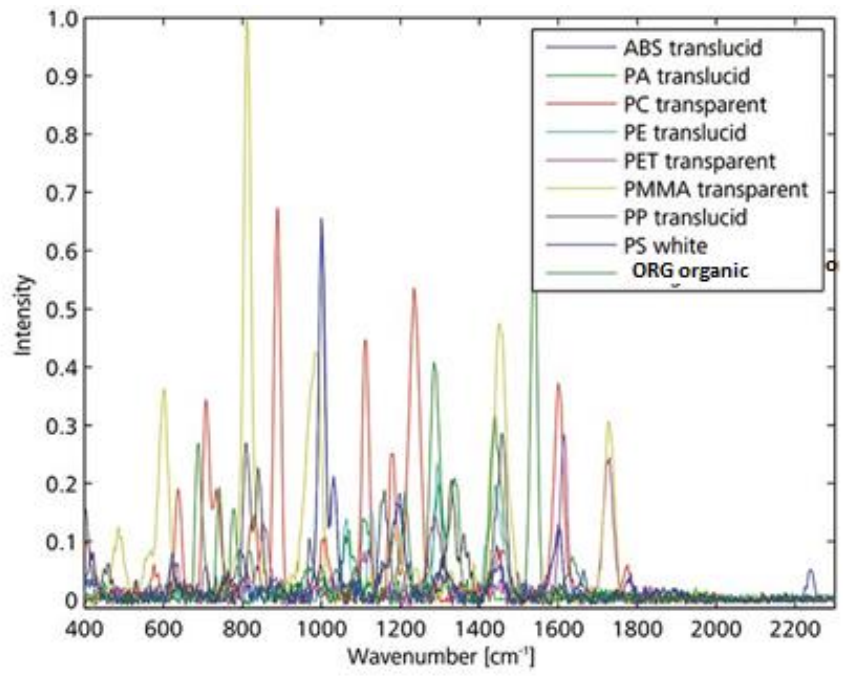

Fuente: Aguiar Santiago. 2020 
La espectroscopia infrarroja es una de las técnicas de espectroscopia molecular, que, junto con la espectrofotometría UV-vis, permiten obtener información estructural de la materia. En esta técnica, un haz de luz infrarroja incide sobre la materia y provoca vibraciones de los átomos de la molécula. Las vibraciones son específicas a determinadas frecuencias de los enlaces químicos, que corresponden a niveles de energía de la molécula, y van a depender de la forma de la superficie de energía potencial de la molécula, la geometría molecular, las masas atómicas y, posiblemente, el acoplamiento vibracional. La cantidad de luz absorbida es registrada continuamente, rango de longitudes de onda de interés, por lo general, 4000-400 cm-1 (Stuart, 2004)

Dado que las bandas vibraciones son características para muchos grupos funcionales, se emplea los espectros como conjunto para identificar a las moléculas.

Los análisis de espectrofotometría de infrarrojo confirmaron los resultados obtenidos. Se pudo observar cómo los espectros característicos de los controles a base de polímeros sintéticos de plásticos con los que se comparó no presentaron cambios en las bandas de absorción características de los enlaces $\mathrm{C}-\mathrm{H}$ y $\mathrm{C}=0$, en ninguno de los experimentos realizados. $\mathrm{Al}$ analizar los espectros de los polisacáridos que contienen las materias primas del tallo de banano, observamos que todos ellos presentan la banda característica del enlace $\mathrm{OH}$, y del enlace $\mathrm{C}-0$ en la región de la huella digital, presentaron, además, una banda de absorción correspondiente al grupo carboxilo $\mathrm{COOH}$, los cuales se utilizaron posteriormente para el análisis cuantitativo. En el grupo de almidón se observó disminución en la absorción de la banda correspondiente al enlace C-0. Para todos los formulados en los que se analizó el grupo funcional característico $\mathrm{COOH}$.

Los resultados obtenidos dejan ver la utilidad que se puede dar a este equipo para continuar realizando investigaciones relacionadas con los polímeros, como efectuar seguimiento a las reacciones de polimerización, caracterizaciones de las estructuras del polímero, y examinar las superficies de los polímeros o los procesos de degradación. Con modelos matemáticos más avanzados y realizando análisis multivariados, es posible, incluso, llegar a proponer modelos para la cuantificación de polímeros en mezclas.

Se obtuvo un material resistente comparado con otros polímeros de los que se elaboran el plástico empleado para la fabricación de utensilios como envases y almacenaje para alimentos. Dado que 
la industria de reciclaje de polímeros está muy poco desarrollada, vale la pena invertir esfuerzos e investigaciones en torno a este tema, ya que estos materiales se convirtieron en uno de los principales problemas de contaminación por la cantidad de residuos de este tipo que son desechados (Rodríguez, Cohen, Ober y Archer, 2014).

\section{Conclusiones}

- En la presente investigación se obtuvo resultados beneficiosos para incursionar en el mercado nacional y extranjeros, con productos de calidad como son los nuestros y por cada parte del tallo nos da como resultado una cantidad de 8 utensilios entre vasos y paltos, el peso neto promedio de los platos obtenidos es de $8.5 \times 10^{-3}$ por $\mathrm{Kg}$ que se traducen a una dimensión de platos de $156 \mathrm{~mm}$ x $14 \mathrm{~mm}$ y peso neto promedio de los vasos de $3.3 \times 10^{-3}$ por $\mathrm{Kg}$ traducido a dimensiones de vasos de $69 \mathrm{~mm}$ x $81 \mathrm{~mm}$ estos con una capacidad de 175 $\mathrm{ml}$.

- Las pruebas realizadas mostraron indicadores aceptables de capacidad de la rotación, finura y resistencia a la deformación y a la humedad con tiempos de degradación muy cortos después de su utilización.

- Con la prueba se espectrofotometría IR se determinó que se obtuvo un material muy resistente comparado con otros polímeros de los que se elaboran el plástico empleado para la fabricación de utensilios como envases y almacenaje para alimentos.

- El aprovechamiento de los tallos de banano, garantiza el aprovechamiento adecuado de residuos que en otras condiciones son fuente de contaminación y proliferación de bacterias que afectan al resto de plantaciones dejando daños irreparables en estas.

- El producto obtenido presento buena acogida por parte de la población, la cual ve en esto una oportunidad de incursionar en un negocio rentable a largo plazo permitiéndoles mejorar su calidad de vida.

- En el mercado nacional como extranjero se ha dado una alternativa viable para la disminución del impacto ambiental causado por plásticos, con la producción de utensilios totalmente biodegradables de un solo uso.

\section{Referencias bibliográficas}

Aldana, S., Vereda, F., Hidalgo Álvarez, R., \& de Vicente, J. (2016). Facile synthesis of magnetic agarose microfibers by directed selfassembly. Polymer, 93, 61-64. 
Bossis, G., Marins, J., Kuzhir, P., Volkova, O., \& Zubarev, A. (2015). Functionalized microfibers for field-responsive materials and biological applications. Journal of Intelligent Material Systems and Structures, 1-9.

Cortés, J., Puig, J., Morales, J., \& Mendizábal, E. (2011). Hidrogeles nanoestructurados termosensibles sintetizados mediante polimerización en microemulsión inversa. Revista Mexicana de Ingeniería Química., 10, 513-520.

Chivrac F, Pollet E, Avérous L. Progress in nano-biocomposites based on polysaccharides and nanoclays. Mater Sci Eng R Reports. 2009; 67; 1-17.

Clave P. Nutrición Humana y Dietética. 2015; 19:153-9.

Conrad U. Polymers from plants to develop biodegradable plastics. Trends Plant Sci. 2005; 10(11):511.

Distancia MDEEA, Factibilidad PDE, La P, Panela PDE, En G. Universidad Nacional de Loja. $2011 ; 22-25$.

Dias, A., Hussain, A., Marcos, A., \& Roque, A. (2011). A biotechnological perspective on the application of iron oxide magnetic colloids modified with polysaccharides. Biotechnology Advances 29, 29, 142-155.

Estrada Guerrero, R., Lemus Torres, D., Mendoza Anaya, D., \& Rodríguez Lugo, V. (2010). Hidrogeles poliméricos potencialmente aplicables en Agricultura. Revista Iberoamericana de Polímeros, 12(2), 76-87.

Song, J., King, S., Yoon, S., Cho, D., \& Jeong, Y. (2014). Enhanced spinnability of narbon nanotube fibers by surfactant addition. Fiberes and Polymers, 15(4), 762-766.

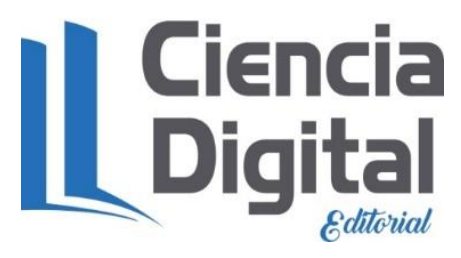




\section{PARA CITAR EL ARTÍCULO INDEXADO}

Aguiar Conya, S. A., García Veloz, M. J., \& Vallejo Abarca, S. M. (2020). Diseño y elaboración de utensilios biodegradables a partir de la fibra del tallo de banano (Musa paradisiaca) como alternativa de uso para mitigar impactos ambientales causados por el plástico . Ciencia Digital, 4(1), 373-384. https://doi.org/10.33262/cienciadigital.v4i1.1118

\section{Ciencia
Digital
Edutaul}

El artículo que se publica es de exclusiva responsabilidad de los autores y no necesariamente reflejan el pensamiento de la Revista Ciencia Digital.

El artículo queda en propiedad de la revista y, por tanto, su publicación parcial y/o total en otro medio tiene que ser autorizado por el director de la Revista Ciencia Digital.
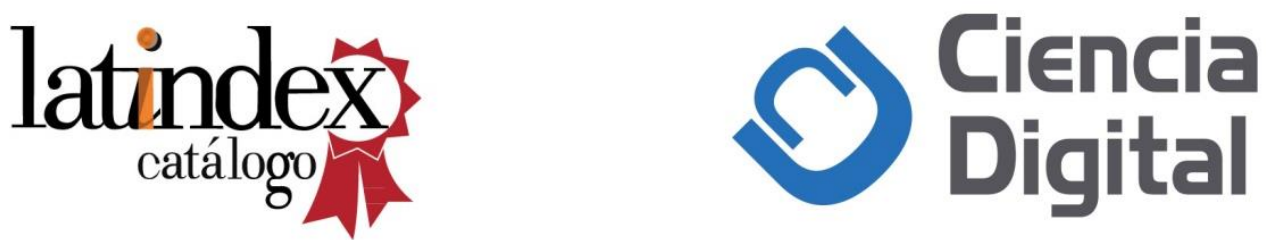